\title{
Preparation and characterization of functional silica hybrid magnetic nanoparticles
}

\author{
Reinaldo G. Digigow ${ }^{\mathrm{a}, \mathrm{b}}$, Jean-François Dechézelles ${ }^{\mathrm{a}}$, Hervé Dietsch ${ }^{\mathrm{a}}$, Isabelle Geissbühler ${ }^{\mathrm{b}}$, \\ Dimitri Vanhecke ${ }^{a}$, Christoph Geers ${ }^{a}$, Ann M. Hirt ${ }^{c}$, Barbara Rothen-Rutishauser ${ }^{a}$, \\ Alke Petri-Fink ${ }^{\mathrm{a}, \mathrm{b}, *}$
}

a Adolphe Merkle Institute, University of Fribourg, Route de l'Ancienne Papeterie CP 209, CH-1723 Marly, Switzerland
b Chemistry Department, University of Fribourg, Chemin du Musée 9, CH-1700 Fribourg, Switzerland

' Institute for Geophysics, ETH Zurich, Sonneggstrasse 5, CH-8092 Zurich, Switzerland

\begin{abstract}
We report on the synthesis and characterization of functional silica hybrid magnetic nanoparticles (SHMNPs). The co-condensation of 3-aminopropyltriethoxysilane (APTES) and tetraethyl orthosilicate (TEOS) in presence of superparamagnetic iron oxide nanoparticles (SPIONs) leads to hybrid magnetic silica particles that are surface-functionalized with primary amino groups. In this work, a comprehensive synthetic study is carried out and completed by a detailed characterization of hybrid particles' size and morphology, surface properties, and magnetic responses using different techniques. Depending on the mass ratio of SPIONs and the two silanes (TEOS and APTES), we were able to adjust the number of surface amino groups and tune the magnetic properties of the superparamagnetic hybrid particles.
\end{abstract}

\section{Introduction}

Superparamagnetic iron oxide nanoparticles (SPIONs) have received a lot of attention for many years because of their outstanding magnetic properties [1,2]. In particular they are used as contrast agents for magnetic resonance imaging (MRI) [3], are investigated in clinical studies for hyperthermia treatment [4,5], or are promising candidates for targeted drug delivery systems [6]. In the field of diagnostics, mostly magnetic separation systems for biomolecules and cells $[7,8]$ have been established in the past and nano-catalytic systems [9] are abundantly investigated for a variety of applications.

Many synthetic approaches are described in the literature but most of the presently reported methods to synthesize SPIONs in an aqueous environment are modifications of the original studies of Massart [10], Bee [11] or Philipse [12] and rely on the co-precipitation of ferrous and ferric iron salts with an aqueous ammonia solution. Such synthesized SPIONs have an isoelectric point, which favors aggregation at physiological $\mathrm{pH}[2,13]$, which alters their magnetic properties [14,15] and impacts biocompatibility [16]. In order to overcome this issue, SPIONs have been coated with surfactants, synthetic polymers, carbohydrates or proteins [17-19]. To prevent agglomeration and to improve chemical stability [20-22], silica is probably the

\footnotetext{
*Corresponding author at: Chemistry Department, University of Fribourg, Chemin du Musée 9, CH-1700 Fribourg, Switzerland.

E-mail address: alke.fink@unifr.ch (A. Petri-Fink).
}

best described inorganic coating material. Silica coating is usually achieved by two synthetic routes: the Stöber [23] process or the microemulsion process [24]. In general, single core-shell particles are obtained by the latter, whereas the Stöber process results in multicore/shell particles [25-27], which are particularly interesting for magnetic separation technologies [28]. Organosilanes are abundantly used for their simplicity of grafting onto SPIONs [3] and, depending on the chemical nature of the functional group, their ability to introduce new ligands and therefore properties to the SPIONs [29,30].

3-Aminopropyltriethoxysilane (APTES) is frequently used to stabilize silica or metal oxide nanoparticles and to introduce amine groups for subsequent chemical functionalization. Since condensation of APTES on SPIONs was reported to result in agglomeration of the particles into small clusters [31], many studies [32] have focused on a two-step approach, where SPIONs are first coated with silica using tetraethyl orthosilicate (TEOS), followed by a second hydrolysis/condensation step using APTES [26,33]. However, up to now, no detailed study about the controlled synthesis of silica hybrid magnetic nanoparticles via co-condensation of APTES and TEOS on SPIONs has been proposed.

In this work we describe the synthesis of functional silica hybrid magnetic nanoparticles (SHMNPs) using SPIONs and a modified Stöber [23] method inspired by the studies of Kobayashi [34] and Takeda [35]. A single-step co-condensation process of APTES and TEOS resulted in silica coating of the SPIONs and the successful incorporation of functional amino groups on the surface 
of the particles, which were characterized in terms of composition (iron, silicon and amine content), size/morphology (transmission electron microscopy and dynamic light scattering), surface properties (zeta potential measurements), and magnetic responses.

\section{Experimental section}

\subsection{Materials}

All chemicals were of analytical reagent grade and were used without any further purification. 3-Aminopropyltriethoxysilane (APTES, 99\%), tetraethyl orthosilicate (TEOS, 99\%), absolute ethanol (99\%), 2,4,6-trinitrobenzene sulfonic acid solution (TNBS, 5.0\%, w/v), iron (III) nitrate nonahydrate (98\%), iron (III) chloride hexahydrate (98\%), iron (II) chloride tetrahydrate (99\%) and sodium dodecyl sulfate $(99 \%)$ were supplied by Sigma-Aldrich. Ammonia $(28 \%$ in water), nitric acid (65\%), and disodium tetraborate decahydrate (98\%), sodium hydroxide (99\%), and sodium bicarbonate (98\%) were purchased from VWR International. Sodium citrate tribasic di-hydrate (99.5\%), glycine (98\%), boric acid (98\%) and ICP-OES standard solutions (silicon and iron, $1000 \mathrm{ppm}$ ) were purchased from Fluka. D-9527 Sigma cellulose membrane dialysis tubing with a molecular weight cutoff of $12 \mathrm{kDa}$ was used for purification. All aqueous solutions were prepared with deionized water obtained from a Milli-Q system (resistivity $=18.2 \mathrm{M} \Omega \mathrm{cm}$, Millipore AG).

\subsection{Synthesis of SPIONs}

SPIONs were prepared by alkaline co-precipitation of ferric and ferrous chlorides in aqueous solution, as previously described $[11,12]$. Typically, $8.6 \mathrm{~g}$ of iron (III) chloride hexahydrate and $3.2 \mathrm{~g}$ of iron (II) chloride tetrahydrate were dissolved in $370 \mathrm{~g}$ of water. Solutions were mixed and precipitated with $30 \mathrm{~mL}$ ammonia. After several washing steps the precipitate was collected and transferred to a reaction flask and $40 \mathrm{~mL}$ nitric acid $(2 \mathrm{M})$ and $60 \mathrm{~mL}$ iron (III) nitrate $(0.27 \mathrm{M})$ were added. The suspension was refluxed for $90 \mathrm{~min}$. The obtained brown suspension of SPIONs was washed again with water, dialyzed against $0.01 \mathrm{M}$ nitric acid for two days, and stored at $4{ }^{\circ} \mathrm{C}$.

\subsection{SPION citrate stabilization}

SPIONs were stabilized by sodium citrate following a method inspired by Khosroshahi et al. [22] and Abramson et al. [36] $9.1 \mathrm{~mL}$ SPIONs suspension (10 mg of $\mathrm{Fe} / \mathrm{ml}$ ) was mixed with $28 \mathrm{~mL}$ sodium citrate $(7.5 \mathrm{mM})$. The temperature was increased to $80^{\circ} \mathrm{C}$ for $30 \mathrm{~min}$ under stirring $(750 \mathrm{rpm})$ and the $\mathrm{pH}$ was adjusted to 7 with an aqueous solution of sodium hydroxide $(0.1 \mathrm{M})$. The suspension of citrate-stabilized SPIONs (3.25 mg of $\mathrm{Fe} / \mathrm{mL}$ ) was stored at $4{ }^{\circ} \mathrm{C}$.

\subsection{Synthesis of silica hybrid magnetic nanoparticles (SHMNPS)}

To synthesize silica hybrid magnetic nanoparticles, we followed a procedure based on the original work reported by Stöber [23] and Barnakov [37]. Typically, $1.45 \mathrm{mg}$ citrate-stabilized SPIONs were diluted in $20 \mathrm{~mL}$ water and mixed with $80 \mathrm{~mL}$ of an ethanolic solution of APTES and TEOS (Table 1). The hydrolysis and co-condensation of TEOS and APTES was initiated by the addition of $1 \mathrm{~mL}$ of ammonia solution (25\%) to the reaction mixture. In this work, $R$ is defined as the SPIONs/silica mass ratio and $R$ was varied by fixing the amount of SPIONs (iron concentration) and adjusting the amount of silica precursors (Table 1). Although different synthetic parameters could have been varied to control the shape and/or shell thickness, we found that best results in terms of reproducibility were obtained by adjusting the APTES and TEOS concentrations. Considering the total reaction, the mass of silica $m_{\text {Silica }}$ is given by Eq. (1). Where $m_{\text {TEOS }}$ is the mass of TEOS, $M_{\text {TEOS }}$ is the molecular weight of TEOS, $m_{\mathrm{APTES}}$ is the mass of APTES, $M_{\mathrm{APTES}}$ is the molecular weight of APTES and $M_{\text {Silica }}$ is the molecular weight of silica.

$m_{\text {Silica }}=\left(\left(m_{\mathrm{TEOS}} / M_{\mathrm{TEOS}}\right)+\left(m_{\mathrm{APTES}} / M_{\mathrm{APTES}}\right)\right) \times M_{\text {Silica }}$

Condensation of the alkoxysilane mixture onto the surface of SPIONs was initiated by adding $1 \mathrm{~mL}$ of ammonia aqueous solution. The reaction occurred under constant stirring and at room temperature for $3 \mathrm{~h}$. The suspension of hybrid particles was centrifuged at 20,000 g for $20 \mathrm{~min}$ to remove all unreacted species. The obtained pellet was re-dispersed in $10 \mathrm{~mL}$ water under sonication for $10 \mathrm{~min}$. This purification step was repeated three times and the final suspensions were stored at $4{ }^{\circ} \mathrm{C}$.

\subsection{Transmission electronic microscopy}

The morphology and the size of the obtained particles were studied by transmission electron microscopy (TEM). Samples were diluted in absolute ethanol $(1: 10)$ and one drop of the diluted suspension was slowly evaporated on a 300 mesh carbon membrane-coated copper grid. TEM experiments were performed on a Philips CM100 Biotwin microscope operated at $80 \mathrm{kV}$. Tomograms were performed on a Tecnai F20 microscope operating at $200 \mathrm{kV}$.

\subsection{Dynamic light scattering}

Dynamic light scattering (DLS) measurements were carried out at $90^{\circ}$ on a photon correlation spectrometer from Brookhaven equipped with a BI-9000AT digital autocorrelator. The concentration was set between 0.030 and $0.100 \mathrm{mg} \mathrm{Fe} / \mathrm{mL}(20 \mathrm{mM}$ borate buffer solution, $\mathrm{pH}=7.5$ ) to avoid multiple light scattering.

\subsection{Zeta potential measurements}

Zeta potential measurements were performed using a Particle Size Analyzer 90 plus from Brookhaven Instruments equipped with a BI9000AT digital autocorrelator and platinum electrode. The electrode was cleaned for $10 \mathrm{~min}$ in an ultrasonic bath prior to each measurement and pre-equilibrated for $2 \mathrm{~min}$ in an aliquot of the sample before acquiring data. The samples were prepared as described above for DLS measurements, i.e. in $20 \mathrm{mM}$ of borate buffer $\mathrm{pH} 7.5$.

\subsection{Vibrating sample magnetometer}

A vibrating-sample magnetometer (VSM, Princeton Measurements Corporation Vibrating Sample Magnetometer - model 3900) was used at room temperature to study the magnetic properties of the SHMNPs in the dried state. Magnetization was measured as a function of applied field up to a maximum field of $796 \mathrm{kA} / \mathrm{m}$, using a averaging time of $100 \mathrm{~ms}$. Prior to the measurement, the samples were centrifuged, the supernatant was discarded, and the pellet was dried at $80^{\circ} \mathrm{C}$ overnight.

\subsection{Inductively coupled plasma optical emission spectrometry}

Quantification of iron and silicon content was performed by inductively coupled plasma optical emission spectrometry (ICPOES) (Optima 7000 DV from Perkin Elmer). Therefore, the samples were dissolved in hydrochloric acid (37\%) and heated for one hour at $85{ }^{\circ} \mathrm{C}$. In a typical procedure, the concentration of iron and silicon was determined from standard solutions (Fe and $\mathrm{Si}$ ) at known concentrations. 
Table 1

Summary of synthetic parameters.

\begin{tabular}{|c|c|c|c|c|c|c|c|}
\hline Name & TEOS (mg) & APTES (mg) & Wt\% APTES & $\begin{array}{l}\text { Theoretical mass of obtained } \\
\text { silica }\left(10^{-2} \mathrm{~g}\right)\end{array}$ & $\begin{array}{l}\text { Number of SPIONs }\left(10^{16}\right) / \text { mass } \\
\text { of silica }(\mathrm{g})\end{array}$ & $\begin{array}{l}\text { mass ratio } \\
R \text { 1:XX }\end{array}$ & $\begin{array}{l}\text { NP/(APTES }+ \text { TEOS }) \\
\left(10^{-15} \mathrm{~mL}^{-1}\right)\end{array}$ \\
\hline A & 160 & 0 & 0 & 4.6 & 1.7 & 32 & 4.5 \\
\hline B & 0 & 170 & 100 & 4.6 & 1.7 & 32 & 4.3 \\
\hline C & 131 & 43 & 25 & 4.9 & 1.6 & 34 & 4.2 \\
\hline D & 173 & 0 & 0 & 4.9 & 1.6 & 35 & 4.2 \\
\hline E & 79 & 28 & 26 & 3.1 & 2.6 & 21 & 6.8 \\
\hline $\mathbf{F}$ & 107 & 0 & 0 & 3.1 & 2.6 & 21 & 6.8 \\
\hline G & 40 & 14 & 26 & 1.5 & 5.0 & 11 & 13.4 \\
\hline H & 56 & 0 & 0 & 1.6 & 4.8 & 11 & 12.9 \\
\hline
\end{tabular}

The average number of SPIONs nanoparticles per mL of APTES + TEOS in SHMNPs samples was estimated from iron measurements.
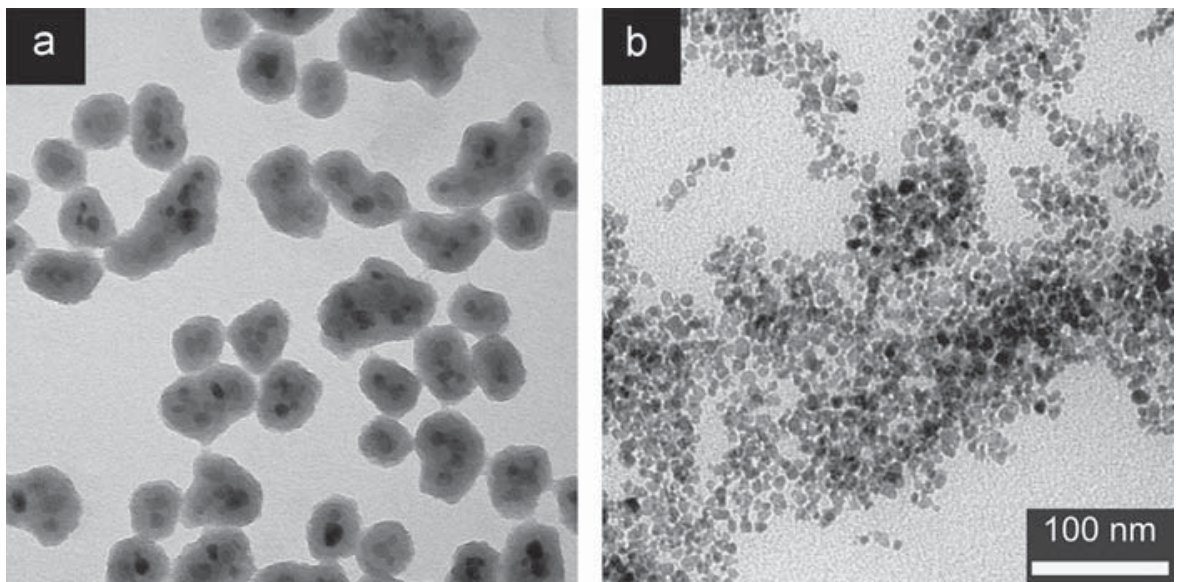

Fig. 1. TEM pictures of SHMNPs synthesized with TEOS only (a) and with APTES only (b). (SPIONs/silica ratio: 1:32). The scale bar applies to all images in the figure.

\subsection{Free amine quantification}

Chemical quantification of primary accessible amines was done according to the Hermanson's method [38]. The colorimetric method is based on the complexation reaction of free amino groups with $0.01 \%(\mathrm{v} / \mathrm{v})$ 2,4,6-trinitrobenzene sulfonic acid solution. Absorption was measured at $335 \mathrm{~nm}$ using a Jasco V-670 Spectrophotometer. The calibration curve was prepared using glycine as standard (SI).

\section{Results and discussion}

In this study, SPIONs were first synthesized in water and subsequently coated with silica through co-condensation of TEOS and APTES to obtain amine-functionalized silica hybrid magnetic nanoparticles (SHMNPs) in a one-step reaction. To investigate the impact of APTES on nanoparticle morphology, surface, and magnetic properties, different APTES/TEOS and SPION/silica ratios were tested.

\subsection{Influence of the silica/SPIONs ratios on morphology}

SPIONs were synthesized by alkaline co-precipitation of ferrous and ferric salts following a previously developed protocol [18]. Typically obtained nanoparticles showed an average diameter of $10 \pm 3 \mathrm{~nm}$, as obtained from TEM (see Fig. SI-1 in supporting information), and agree well with reported data [10-12]. The particles were subsequently stabilized with citrate to avoid flocculation during the silica coating [36]. As shown by Mohammad-Beigi et al. [39], citrate ligands do not perturb the silica condensation onto the SPIONs.
Bare silica particles or silica hybrid particles (i.e. core-shell particles with a silica shell and a different, for example metal core) are generally obtained following the well-known Stöber method based on the hydrolysis and condensation of -*TEOS at basic pH [34,40-45]. Two synthetic pathways are commonly used to graft APTES onto silica particles. The first relies on post-functionalization of pre-formed NPs [46], whereas the second exploits the chemical functionality of APTES by co-condensating APTES and TEOS during nanoparticle synthesis [34]. Following the co-condensation approach and in order to develop a simple one-step method, APTES was mixed with TEOS at different ratios in presence of SPIONs. Two controls using either pure TEOS or APTES were synthesized to define the boundary conditions of the experimental design. Fig. $1 \mathrm{a}$ and $\mathrm{b}$ shows representative transmission electron micrographs of the control nanoparticles synthesized to define the boundary conditions of the experimental design. It was previously shown by van Blaaderen et al. [47] that small silica particles are usually less monodispersed and less spherical, and that the surface roughness is higher compared to larger silica NPs, which are almost perfectly smooth and monodispersed spheres. This is confirmed in Fig. 1a showing SPIONs incorporated in non-spherical silica particles. This shape anisotropy might be explained by the presence of small pre-formed SPIONs@SiO 2 aggregates, which is confirmed by dynamic light scattering experiments, showing a mean hydrodynamic diameter of around $70 \mathrm{~nm}$, which cannot be attributed to single core-shell NPs. Philipse et al. [25] have shown that some clusters of SPIONs and silica are formed in the beginning of the silica condensation and determine the final shape of the hybrid particles. Such aggregation partially explains the bead like character of the final materials as compared to single core-shell NPs (Fig. 2a). Fig. 1b shows a micrograph of the structure obtained using pure APTES together with SPIONs. In agreement with van Blaaderen et al. [47], a gel-like structure consisting of very small aggregates was detected 


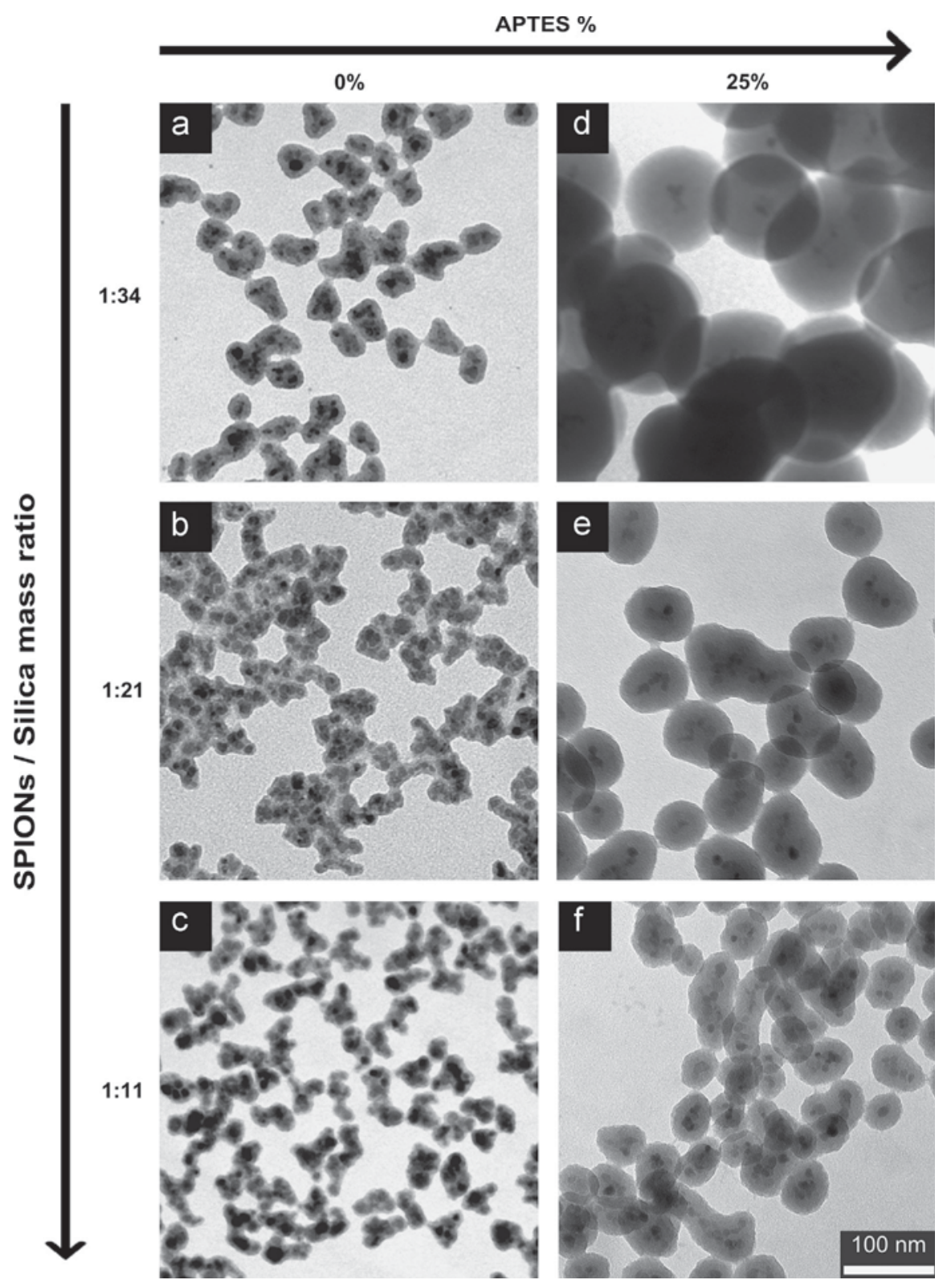

Fig. 2. TEM pictures of SHMNPs synthesized from different APTES mass ratio (from left to right) and different SPIONS / silica amount: 0 wt\% APTES (a-c), 25 wt\% APTES (d-f). SPIONS/Silica ratio: 1:34 ( $\mathrm{a}$ and b), 1:21 ( $\mathrm{b}$ and e), 1:11 ( $\mathrm{c}$ and $\mathrm{f}$ ). The scale bar applies to all images in the figure.

and virtually no silica coating of the SPIONs was observed. Nevertheless, a thin layer of APTES might have been grafted on the particles $[3,31,45]$. This morphology could be attributed to the aminopropyl-group in APTES, because compared to tetra-alkoxysilanes such as TEOS, trialkoxysilanes such as APTES show a lower cross-linking density in the gel network $[48,49]$. It was previously shown by Chen et al. [50] that the hydrolyzed APTES molecule forms a six- or five-membered chelate ring, which sterically hampers condensation. Chen et al. [50] also demonstrated that at basic $\mathrm{pH}$, the terminal protonated amine $\left(-\mathrm{NH}_{3}^{+}\right)$group is prone to hydrogen bonding to the $\mathrm{Si}-\mathrm{O}^{-}$group of a second APTES molecule. This bond might be strong enough to avoid condensation with other APTES molecules, which was confirmed by a previous study [51].

To study the size and morphology of the hybrid magnetic nanoparticles, we varied the (a) concentration of APTES in TEOS ( 0 and $25 \mathrm{wt} \%$ ) and (b) $R$, which was previously defined as the SPIONs/silica mass ratio (1:11, $1: 21$ and 1:34). The addition of APTES to TEOS while maintaining the SPIONs/silica ratio (Fig. 2d) resulted in smooth, slightly larger, and less anisotropic particles.
The co-condensation of APTES and TEOS leads to the formation of a multiple SPIONs core - silica shell architecture as illustrated by the tomogram (Fig. 3). Note that the SPIONs are incorporated in the silica matrix and are not merely adsorbed to the silica sphere. As a general trend we observed a (i) rougher surface, (ii) higher anisotropy, (iii) increased number of SPIONs incorporated in the silica beads, and (iv) a thinner silica shell with decreasing $R$ (Fig. 2d-f).

Surface roughness had been previously related to particle size, in the sense that the roughness of such materials increased with decreasing particle size [47]. The large number of incorporated SPIONs per silica bead (see tomogram in Fig. 3) might be attributed to the formation of SPION aggregates during the synthesis, as already described above. However, the presence of APTES can prevent or hamper aggregation of primary particles, which is in line with our findings. Fig. 2 indicates a higher number of incorporated and aggregated SPIONs in batches without APTES (compare to Fig. 1) [48]. Another factor might be the comparably smaller silane amount, which is not enough to efficiently form and 

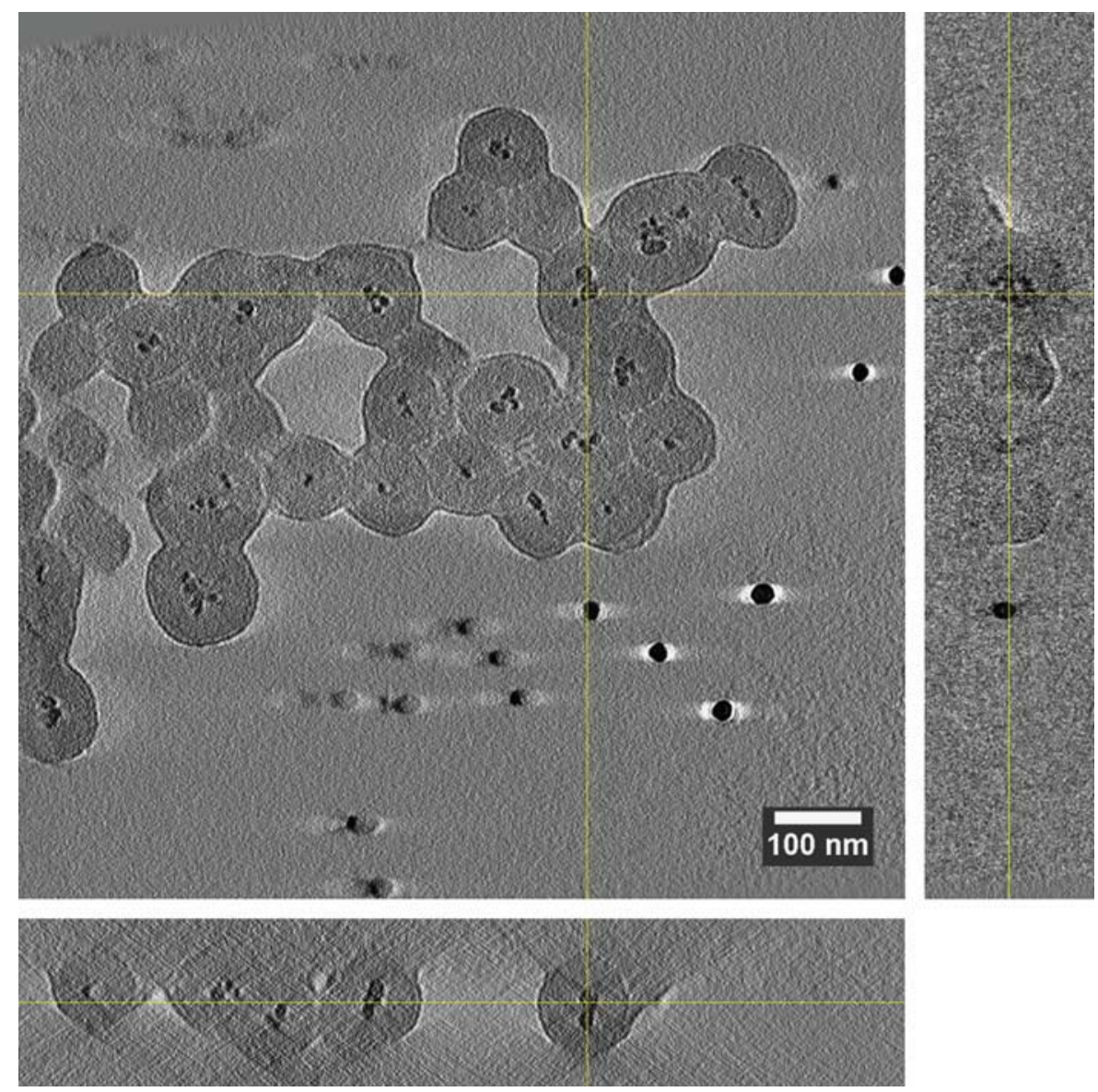

Fig. 3. Electron tomogram of SHMNPs (SPIONs/silica ratio: $1: 21,14$ wt\% APTES). The sample holder was tilted between -60 and $+60^{\circ}$, with a $2^{\circ}$ increment. The picture shows the $x y$ stacks with representative side views in $x z$ (bottom) and $y z$ (right) direction slices from the three dimensional stacks.

stabilize single core shell nanoparticles. As described by Almeida et al. [26], the hydrolyzed and partially condensed silane molecules deposit on the individual or aggregated SPIONs. These small hybrid particles aggregate randomly at low TEOS concentration and so, a uniform growth of silica cannot occur.

In agreement with a recently published study by Kong et al. [52], dynamic light scattering measurements confirmed (Fig. 4) the increase of the apparent hydrodynamic diameter with increasing APTES concentration in the silane precursor mixture. The large error bars in Fig. 4 might be explained by the polydispersity of the sample and indirectly confirms the presence of aggregates formed in the presence of the aminopropyl chain, which increases the particles' hydrophobicity thus inducing flocculation (see Fig. SI-2, supporting information) [53-56].

\subsection{Surface properties}

In order to tune the surface properties of the produced SHMNPs, we varied the amount of APTES in the co-condensation process. Fig. 5 shows the zeta potential of the particles as function of APTES concentration for three SPIONs/silica ratios $R$.

As expected, SHMNPs showed negative zeta potential values $(-20<\xi<-50 \mathrm{mV})$ in the absence of APTES, which is in agreement with previous studies $[57,58]$. Above the isoelectric point of silica $(\mathrm{pH}>2)$, the silica surface is negatively charged because of the presence of deprotonated silanol groups $\left(\mathrm{SiO}^{-}\right)$[44].

Upon addition of APTES, the zeta potential increases, which is attributed to the increasing number of protonated amine $\left(-\mathrm{NH}_{3}^{+}\right)$ groups $(\mathrm{pKa}=9)$ [59] on the nanoparticle surface. The zeta potential measurements of samples made with 10\% APTES showed

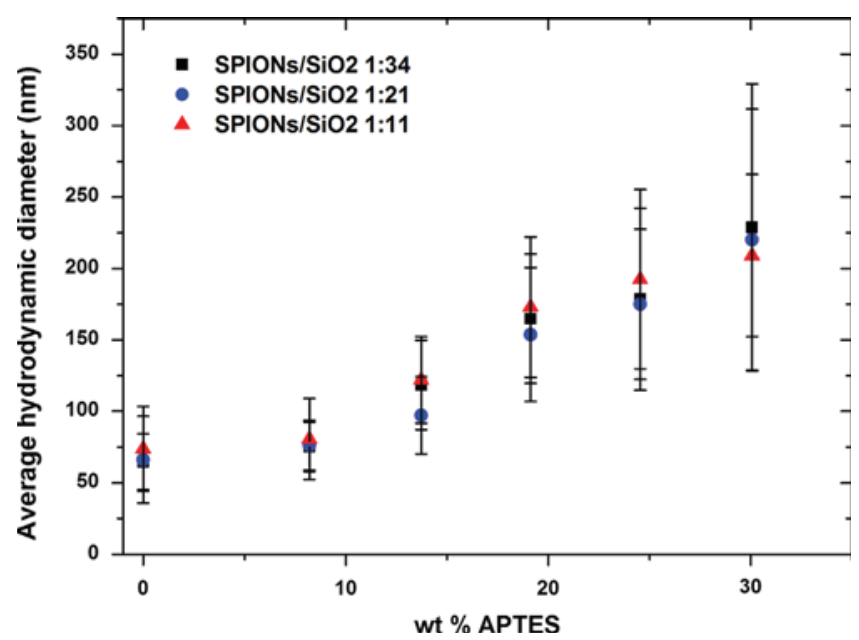

Fig. 4. APTES mass ratio dependence of the hydrodynamic diameter of the SHMNPs as measured by DLS. The error bars represent the width of the population curve.

a neutral value, which could come from a charge compensation with positive charges of the amino groups. When adding more APTES, a net positive surface charge appears. At higher APTES concentration, the increase in zeta potential is less prominent, suggesting a saturation of the particles' surfaces with $\left(-\mathrm{NH}_{3}^{+}\right)$ groups. Moreover, SHMNPs grafted with large amounts of APTES are less stable in water (see Fig. SI-2, supporting information).

In the next step, we determined the total number of primary amino groups available on the silica surface using a previously 


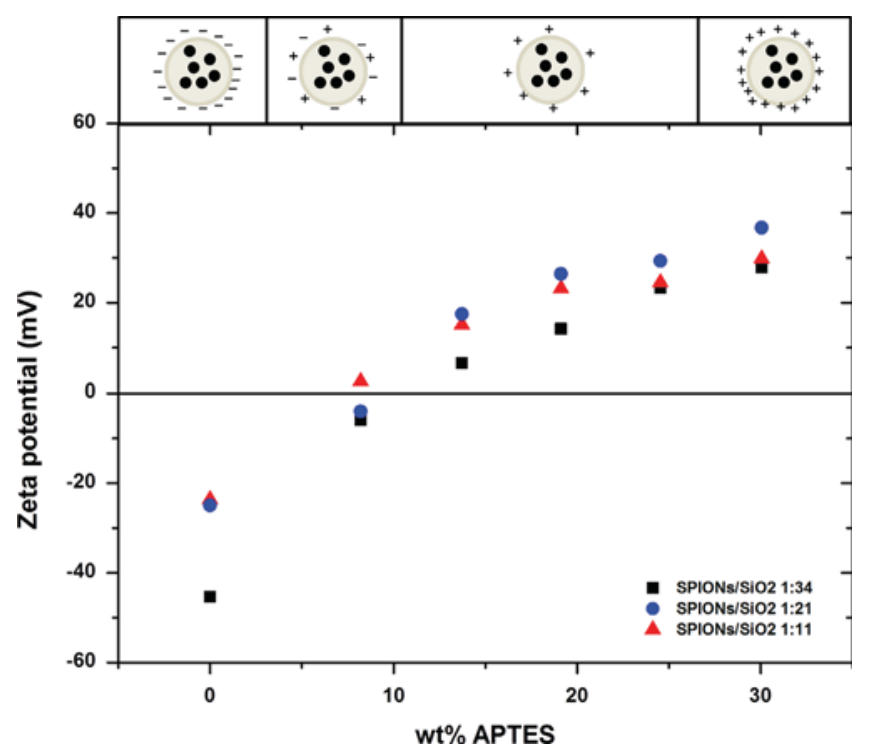

Fig. 5. Zeta potential of SHMNPs as function of the APTES concentration at fixed $\mathrm{pH}=7.5$ (in $20 \mathrm{mM}$ of borate buffer). Top: schematic model of the charges present on the surface of the SHMNPs as function of APTES concentration.

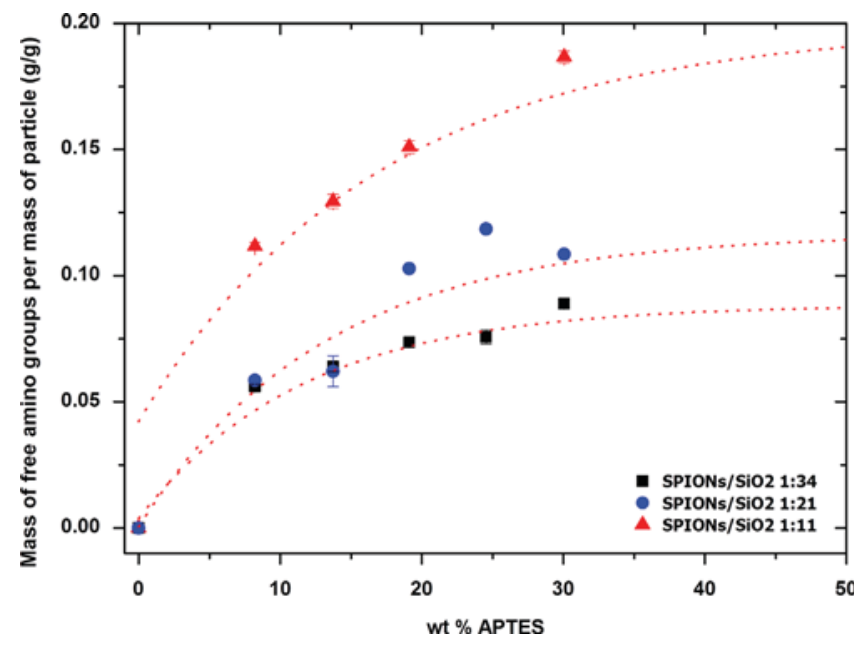

Fig. 6. Evolution of the mass of free amino groups per mass of SHMNPs as a function of the APTES mass ratio determined by colorimetric assay. Lines serve as guide for the eyes.

a

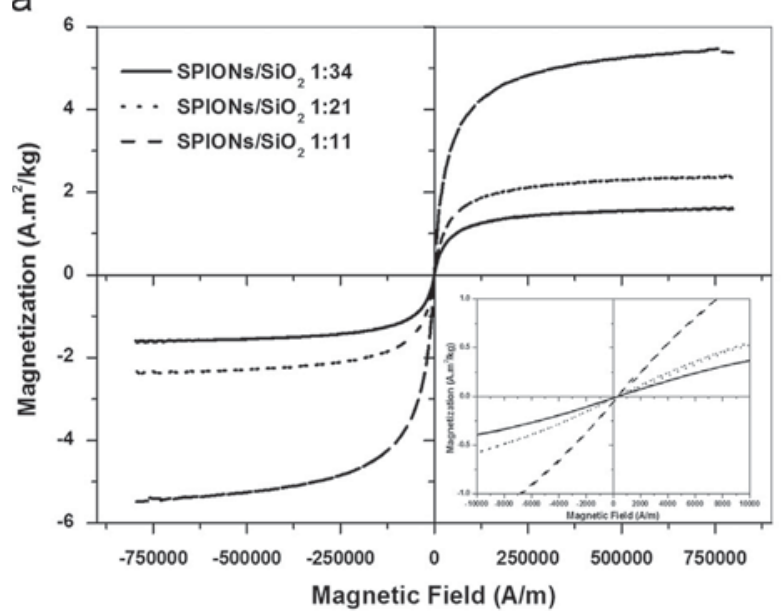

validated colorimetric assay [60]. Fig. 6 shows the correlation between the number of amino groups vs. APTES concentration for three SPION/silica ratios. As expected, the number of accessible amino group increases with increasing APTES concentration which confirms the previous result.

\subsection{Magnetic properties}

The magnetization of SHMNPs was measured as function of the magnetic field using VSM at room temperature (Fig. 7a). No hysteresis was detected confirming the superparamagnetic behavior of the particles, i.e., samples carry no remanent magnetization and have no coercivity (Fig. 7a). The saturation magnetization increases with increasing SPIONs $/ \mathrm{SiO}_{2}$ ratio, indicating that a thicker silica shell is accompanied by a lower magnetization of the particles. This has been shown before; when the introduction of a non-magnetic material shell (i.e. silica) has led to a dilution of the SPIONs magnetic behavior due to the additional mass of the silica shell [61]. Taking into account the saturation magnetization of bare, i.e. uncoated SPIONs (Fig. SI-3 in support information) and SHMNPs (Fig. 7a) at fixed field value (i.e. $398 \mathrm{kA} / \mathrm{m}$ ) (Eq. (2)), we estimated the SPIONs/silica mass ratio in the final synthesis process. From these numerical estimations, we found theoretical ratios of $1: 36,1: 24$, and $1: 11$ for the respective experimental ratios of $1: 34,1: 21$ and $1: 11$ respectively.

Mass ratio $=\left(\frac{\mathrm{MH}_{\text {naked SPIONs, } 398 \mathrm{kA} / m}}{\mathrm{MH}_{\text {hybrid particle, } 398 \mathrm{kA} / \mathrm{m}}}\right)$

We determined the susceptibility as function of the experimental SPIONs/silica mass ratio (Fig. 7b). Susceptibility was calculated from the slope of the fitted curve in the linear $\mathrm{MH}$ dependence at low field (up to $4000 \mathrm{~A} / \mathrm{m}$, inset of Fig. 7a). We showed that the susceptibility linearly increases with increasing amount of SPIONs [32].

\section{Conclusion}

We report the synthesis of functional silica hybrid magnetic nanoparticles by a single-step co-condensation process of APTES and TEOS in presence of SPIONs. This one-pot reaction leads to hybrid magnetic silica particles whose surface is functionalized with primary amino groups. In this work, we carried out a detailed characterization of the hybrid particles in terms of composition

b

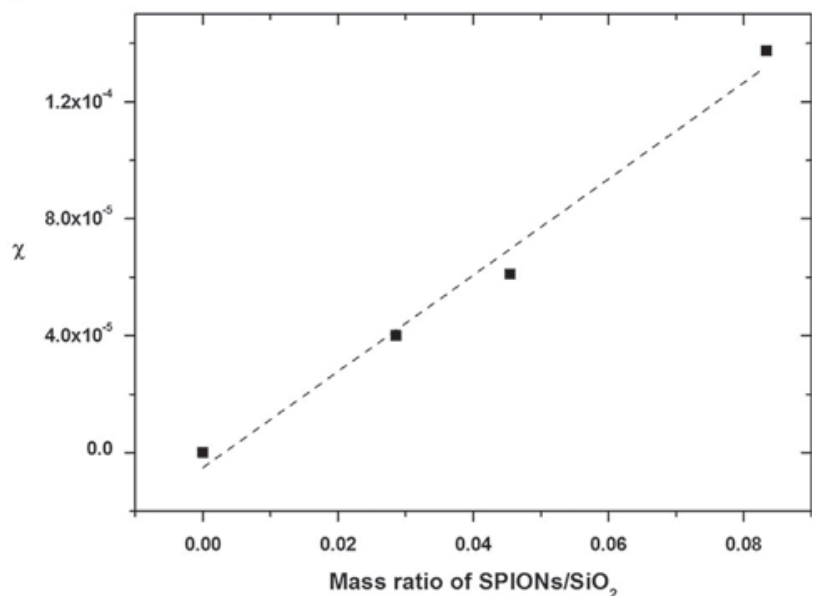

Fig. 7. (a) Magnetization $M H$ curves of the SHMNPs synthesized with different SPIONs/silica ratios. Inset shows a magnified plot near $H=0$. (b) Magnetic susceptibility as a function of the mass ratio of SPIONs/SiO 2 . 
(iron, silicon and amine content), size/morphology (transmission electron microscopy and dynamic light scattering), surface properties (zeta potential measurements), and magnetic responses. By tuning the mass ratio of SPIONs and silanes, respectively, we could adjust the number of surface amino groups and control the magnetic properties of the hybrid particles, whose superparamagnetic properties were preserved in the process.

\section{Acknowledgment}

The authors gratefully acknowledge financial support from the Swiss National Science Foundation (Project 123373), the University of Fribourg, Fribourg Center for Nanomaterials (FriMat) and the Adolphe Merkle Foundation.

\section{Appendix A. Supporting information}

Supplementary data associated with this article can be found in the online version

\section{References}

[1] S. Bedanta, W. Kleemann, Supermagnetism, J. Phys. D: Appl. Phys. 42 (2009) $1-28$.

[2] M.C. Tan, G. Chow, Nanostructured Materials for Biomedical Applications, Transworld Research Network, Kerala, 2009.

[3] C. Zhang, B. Wängler, B. Morgenstern, H. Zentgraf, M. Eisenhut, H. Untenecker, R. Krüger, R. Huss, C. Seliger, W. Semmler, F. Kiessling, Silica- and alkoxysilanecoated ultrasmall superparamagnetic iron oxide particles: a promising tool to label cells for magnetic resonance imaging, Langmuir 23 (2007) 1427-1434.

[4] S.L. Ho, L. Jian, W. Gong, W.N. Fu, Design and analysis of a novel targeted magnetic fluid hyperthermia system for tumor treatment, IEEE Trans. Magn. 48 (2012) 3262-3265.

[5] Z. Li, K. Kawamura, M. Kawashita, T.-A. Kudo, H. Kanetaka, M. Hiraoka, in vitro assessment of poly(methylmethacrylate)-based bone cement containing magnetite nanoparticles for hyperthermia treatment of bone tumor, J. Biomed. Mater. Res. 100A (2012) 2537-2545.

[6] C. Alexiou, R.J. Schmid, R. Jurgons, M. Kremer, G. Wanner, C. Bergemann, E. Huenges, T. Nawroth, W. Arnold, F.G. Parak, Targeting cancer cells: magnetic nanoparticles as drug carriers, Eur. Biophys. J. 35 (2006) 446-450.

[7] Y. Okamoto, F. Kitagawa, K. Otsuka, Online concentration and affinity separation of biomolecules using multifunctional particles in capillary electrophoresis under magnetic field, Anal. Chem. 79 (2007) 3041-3047.

[8] K. Ni, H. Lu, C. Wang, K.C. Black, D. Wei, Y. Ren, P.B. Messersmith, A novel technique for in situ aggregation of Gluconobacter oxydans using bio-adhesive magnetic nanoparticles, Biotechnol. Bioeng. 109 (2012) 2970-2977.

[9] M.M. Mojtahedi, M.S. Abaee, A. Rajabi, P. Mahmoodi, S. Bagherpoor, Recyclable superparamagnetic $\mathrm{Fe}_{3} \mathrm{O}_{4}$ nanoparticles for efficient catalysis of thiolysis of epoxides, J. Mol. Catal. A: Chem. 361-362 (2012) 68-71.

[10] R. Massart, Preparation of aqueous magnetic liquids in alkaline and acidic media, IEEE Trans. Magn. 17 (1981) 1247-1248.

[11] A. Bee, R. Massart, S. Neveu, Synthesis of very fine maghemite particles, J. Magn. Magn. Mater. 149 (1995) 6-9.

[12] G. van Ewijk, G. Vroege, A. Philipse, Convenient preparation methods for magnetic colloids, J. Magn. Magn. Mater. 201 (1999) 31-33.

[13] M. Mikhaylova, D.K. Kim, C.C. Berry, A. Zagorodni, M. Toprak, A.S.G. Curtis, M. Muhammed, BSA immobilization on amine-functionalized superparamagnetic iron oxide nanoparticles, Chem. Mater. 16 (2004) 2344-2354.

[14] D. Eberbeck, C.L. Dennis, N.F. Huls, K.L. Krycka, C. Gruttner, F. Westphal, Multicore magnetic nanoparticles for magnetic particle imaging, IEEE Trans. Magn. 49 (2013) 269-274.

[15] H. Pardoe, W. Chua-anusorn, T.G. St. Pierre, J. Dobson, Structural and magnetic properties of nanoscale iron oxide particles synthesized in the presence of dextran or polyvinyl alcohol, J. Magn. Magn. Mater. 225 (2001) 41-46.

[16] A. Figuerola, R. Di Corato, L. Manna, T. Pellegrino, From iron oxide nanoparticles towards advanced iron-based inorganic materials designed for biomedical applications, Pharmacol. Res. 62 (2010) 126-143.

[17] D. Kim, Y. Zhang, W. Voit, K. Rao, M. Muhammed, Synthesis and characterization of surfactant-coated superparamagnetic monodispersed iron oxide nanoparticles, J. Magn. Magn. Mater. 225 (2001) 30-36.

[18] M. Chastellain, A. Petri, H. Hofmann, Particle size investigations of a multistep synthesis of PVA coated superparamagnetic nanoparticles, J. Colloid Interface Sci. 278 (2004) 353-360.

[19] D. Ma, T. Veres, L. Clime, F. Normandin, J. Guan, D. Kingston, B. Simard, Superparamagnetic $\mathrm{Fe}_{x} \mathrm{O}_{y} @ \mathrm{SiO}_{2}$ core-shell nanostructures: controlled synthesis and magnetic characterization, J. Phys. Chem. C 111 (2007) 1999-2007.
[20] Y. Lu, Y. Yin, B.T. Mayers, Y. Xia, Modifying the Surface properties of superparamagnetic iron oxide nanoparticles through a sol-gel approach, Nano Lett. 2 (2002) 183-186.

[21] Y. Deng, C. Wang, J. Hu, W. Yang, S. Fu, Investigation of formation of silicacoated magnetite nanoparticles via sol-gel approach, Colloids Surf. A 262 (2005) 87-93.

[22] M. Khosroshahi, L. Ghazanfari, Preparation and characterization of silica-coated iron-oxide bionanoparticles under $\mathrm{N}_{2}$ gas, Physica E 42 (2010) 1824-1829.

[23] W. Stöber, A. Fink, Controlled growth of monodisperse silica spheres in the micron size range, J. Colloid Interface Sci. 26 (1968) 62-69.

[24] H.L. Ding, Y.X. Zhang, S. Wang, J.M. Xu, S.C. Xu, G.H. Li, $\mathrm{Fe}_{3} \mathrm{O}_{4} @ \mathrm{SiO}_{2}$ core/shell nanoparticles: the silica coating regulations with a single core for different core sizes and shell thicknesses, Chem. Mater. 24 (2012) 4572-4580.

[25] A.P. Philipse, M.P.B. van Bruggen, C. Pathmamanoharan, Magnetic silica dispersions: preparation and stability of surface-modified silica particles with a magnetic core, Langmuir 10 (1994) 92-99.

[26] M. Almeida, K. Caiado, P. Sartoratto, D. Cintra e Silva, A. Pereira, P. Morais, Preparation and size-modulation of silica-coated maghemite nanoparticles, J. Alloys Compd. 500 (2010) 149-152.

[27] R. Arenal, L. de Matteis, L. Custardoy, A. Mayoral, M. Tence, V. Grazu, J.M. de La Fuente, C. Marquina, M.R. Ibarra, Spatially-resolved EELS analysis of antibody distribution on biofunctionalized magnetic nanoparticles, ACS Nano 7 (2013) 4006-4013.

[28] S. Berensmeier, Magnetic particles for the separation and purification of nucleic acids, Appl. Microbiol. Biotechnol. 73 (2006) 495-504.

[29] J. Jang, H. Lim, Characterization and analytical application of surface modified magnetic nanoparticles, Microchem. J. 94 (2010) 148-158.

[30] N. Frickel, R. Messing, T. Gelbrich, A.M. Schmidt, Functional silanes as surface modifying primers for the preparation of highly stable and well-defined magnetic polymer hybrids, Langmuir 26 (2010) 2839-2846.

[31] S. Mohapatra, N. Pramanik, S. Mukherjee, S.K. Ghosh, P. Pramanik, A simple synthesis of amine-derivatised superparamagnetic iron oxide nanoparticles for bioapplications, J. Mater. Sci. 42 (2007) 7566-7574.

[32] C. Vogt, M.S. Toprak, M. Muhammed, S. Laurent, J.L. Bridot, R.N. Müller, High quality and tuneable silica shell-magnetic core nanoparticles, J. Nanopart. Res. 12 (2010) 1137-1147.

[33] K. Kang, J. Choi, J.H. Nam, S.C. Lee, K.J. Kim, S.W. Lee, J.H. Chang, Preparation and characterization of chemically functionalized silica-coated magnetic nanoparticles as a DNA separator, J. Phys. Chem. B 113 (2009) $536-543$.

[34] Y. Kobayashi, M. Horie, M. Konno, B. Rodríguez-González, L.M. Liz-Marzán, Preparation and properties of silica-coated cobalt nanoparticles, J. Phys. Chem. B 107 (2003) 7420-7425.

[35] Y. Takeda, Y. Komori, H. Yoshitake, Direct Stöber synthesis of monodisperse silica particles functionalized with mercapto-, vinyl- and aminopropylsilanes in alcohol-water mixed solvents, Colloids Surf. A: Physicochem. Eng. Asp. 422 (2013) 68-74.

[36] S. Abramson, L. Srithammavanh, J.M. Siaugue, O. Horner, X. Xu, V. Cabuil, Nanometric core-shell-shell $\gamma-\mathrm{Fe}_{2} \mathrm{O}_{3} / \mathrm{SiO}_{2} / \mathrm{TiO}_{2}$ particles, J. Nanopart. Res. 11 (2009) 459-465

[37] Y.A. Barnakov, M.H. Yu, Z. Rosenzweig, Manipulation of the magnetic properties of magnetite - silica nanocomposite materials by controlled Stöber synthesis, Langmuir 21 (2005) 7524-7527.

[38] G.T. Hermanson, Bioconjugate techniques, second ed., Academic Press, Amsterdam [Netherlands], 2008 (Boston [Mass.])

[39] H. Mohammad-Beigi, S. Yaghmaei, R. Roostaazad, H. Bardania, A. Arpanaei, Effect of $\mathrm{pH}$, citrate treatment and silane-coupling agent concentration on the magnetic, structural and surface properties of functionalized silica-coated iron oxide nanocomposite particles, Physica E 44 (2011) 618-627.

[40] F. Caruso, Nanoengineering of particle surfaces, Adv. Mater. 13 (2001) 11-22.

41] Y. Kobayashi, H. Katakami, E. Mine, D. Nagao, M. Konno, L.M. Liz-Marzán, Silica coating of silver nanoparticles using a modified Stöber method, J. Colloid Interface Sci. 283 (2005) 392-396.

[42] A.F. Demirörs, A. van Blaaderen, A. Imhof, Synthesis of eccentric titania-silica core-shell and composite particles, Chem. Mater. 21 (2009) 979-984.

[43] J.-F. Dechézelles, G. Mialon, T. Gacoin, C. Barthou, C. Schwob, A. Maître, R. Vallée, H. Cramail, S. Ravaine, Inhibition and exaltation of emission in layer-controlled colloidal photonic architectures, Colloids Surf. A: Physicochem. Eng. Asp. 373 (2011) 1-5.

[44] J.-F. Dechézelles, V. Malik, J. Crassous, P. Scurtenberger, Hybrid raspberry microgels with tunable thermoresponsive behavior, Soft Matter 9 (2013) 2798-2802.

[45] M. Yamaura, Preparation and characterization of (3-aminopropyl)triethoxysilanecoated magnetite nanoparticles, J. Magn. Magn. Mater. 279 (2004) 210-217.

[46] S.L. Westcott, S.J. Oldenburg, T.R. Lee, N.J. Halas, Formation and adsorption of clusters of gold nanoparticles onto functionalized silica nanoparticle surfaces, Langmuir 14 (1998) 5396-5401.

[47] A. van Blaaderen, A. Vrij, Synthesis and characterization of monodisperse organo-silica spheres, J. Colloid Interface Sci. 156 (1993) 1-18.

[48] I. Rahman, M. Jafarzadeh, C. Sipaut, Synthesis of organo-functionalized nanosilica via a co-condensation modification using $\gamma$-aminopropyltriethoxysilane (APTES), Ceram. Int. 35 (2009) 1883-1888.

[49] H. Schmidt, H. Scholze, A. Kaiser, Principles of hydrolysis and condensation reaction of alkoxysilanes, J. Non-Cryst. Solids 63 (1984) 1-11.

[50] S. Chen, S. Hayakawa, Y. Shirosaki, E. Fujii, K. Kawabata, K. Tsuru, A. Osaka, Sol-gel synthesis and microstructure analysis of amino-modified hybrid silica 
nanoparticles from aminopropyltriethoxysilane and tetraethoxysilane, J. Am. Ceram. Soc. 92 (2009) 2074-2082.

[51] A.M. Jakob, T.A. Schmedake, A. Novel, Approach to monodisperse, luminescent silica spheres, Chem. Mater. 18 (2006) 3173-3175.

[52] D. Kong, C. Zhang, Z. Xu, G. Li, Z. Hou, J. Lin, Tunable photoluminescence in monodisperse silica spheres, J. Colloid Interface Sci. 352 (2010) 278-284.

[53] T. Jesionowski, A. Krysztafkiewicz, Preparation of the hydrophilic/hydrophobic silica particles, Colloids Surf. A: Physicochem. Eng. Asp. 207 (2002) 49-58.

[54] S.A. Kulkarni, S.B. Ogale, K.P. Vijayamohanan, Tuning the hydrophobic properties of silica particles by surface silanization using mixed self-assembled monolayers, J. Colloid Interface Sci. 318 (2008) 372-379.

[55] A. Krysztafkiewicz, T. Jesionowski, S. Binkowski, Precipitated silicas modified with 3-aminopropyltriethoxysilane, Colloids Surf. A: Physicochem. Eng. Asp. 173 (2000) 73-84.

[56] T. Jesionowski, A. Krysztafkiewicz, Influence of silane coupling agents on surface properties of precipitated silicas, Appl. Surf. Sci. 172 (2001) 18-32.
[57] J.M. Rosenholm, M. Lindén, Wet-chemical analysis of surface concentration of accessible groups on different amino-functionalized mesoporous SBA-15 silicas, Chem. Mater. 19 (2007) 5023-5034.

[58] X.-K. Ma, N.-H. Lee, H.-J. Oh, J.-W. Kim, C.-K. Rhee, K.-S. Park, S.-J. Kim, Surface modification and characterization of highly dispersed silica nanoparticles by a cationic surfactant, Colloids Surf. A: Physicochem. Eng. Asp. 358 (2010) $172-176$.

[59] T. Ung, L.M. Liz-Marzán, P. Mulvaney, Controlled method for silica coating of silver colloids. Influence of coating on the rate of chemical reactions, Langmuir 14 (1998) 3740-3748.

[60] N. Griffete, M.J. Clift, A. Lamouri, R.G. Digigow, A.M. Mihut, A. Fink, B. RothenRutishauser, H. Dietsch, Amino covalent binding approach on iron oxide nanoparticle surface: toward biological applications, Colloids Surf. A: Physicochem. Eng. Asp. 415 (2012) 98-104.

[61] S. Kralj, D. Makovec, S. Campelj, M. Drofenik, Producing ultra-thin silica coatings on iron-oxide nanoparticles to improve their surface reactivity, J. Magn. Magn. Mater. 322 (2010) 1847-1853. 